\title{
Well-being of nursing staff on specialized units for older patients with combined care needs
}

Citation for published version (APA):

Collet, J., de Vugt, M. E., Schols, J. M. G. A., Engelen, G. J. J. A., Winkens, B., \& Verhey, F. R. J. (2018). Well-being of nursing staff on specialized units for older patients with combined care needs. Journal of Psychiatric and Mental Health Nursing, 25(2), 108-118. https://doi.org/10.1111/jpm.12445

Document status and date:

Published: 01/03/2018

DOI:

10.1111/jpm. 12445

Document Version:

Publisher's PDF, also known as Version of record

Document license:

Taverne

Please check the document version of this publication:

- A submitted manuscript is the version of the article upon submission and before peer-review. There can be important differences between the submitted version and the official published version of record.

People interested in the research are advised to contact the author for the final version of the publication, or visit the DOI to the publisher's website.

- The final author version and the galley proof are versions of the publication after peer review.

- The final published version features the final layout of the paper including the volume, issue and page numbers.

Link to publication

\footnotetext{
General rights rights.

- You may freely distribute the URL identifying the publication in the public portal. please follow below link for the End User Agreement:

www.umlib.nl/taverne-license

Take down policy

If you believe that this document breaches copyright please contact us at:

repository@maastrichtuniversity.nl

providing details and we will investigate your claim.
}

Copyright and moral rights for the publications made accessible in the public portal are retained by the authors and/or other copyright owners and it is a condition of accessing publications that users recognise and abide by the legal requirements associated with these

- Users may download and print one copy of any publication from the public portal for the purpose of private study or research.

- You may not further distribute the material or use it for any profit-making activity or commercial gain

If the publication is distributed under the terms of Article $25 \mathrm{fa}$ of the Dutch Copyright Act, indicated by the "Taverne" license above, 


\title{
Well-being of nursing staff on specialized units for older patients with combined care needs
}

\author{
J. Collet MSc, Physician, PhD Canditate ${ }^{1,2, *}$ (D) | M. E. de Vugt Professor, Healthcare \\ Psychologist $^{3}$ | J. M. G. A. Schols Professor, Physician ${ }^{1}$ | G. J. J. A. Engelen MSc, Director, \\ Clinical Psychologist $^{2}$ | B. Winkens PhD, Statistician ${ }^{4}$ | F. R. J. Verhey Professor, \\ Neuropsychiatrist $^{3}$
}

\begin{abstract}
${ }^{1}$ Faculty of Health, Medicine and Life Sciences (FHML), Care and Public Health Research Institute (CAPHRI), Department of Family Medicine/Elderly Care Medicine and Department of Health Services Research, Maastricht University, Maastricht, Netherlands

${ }^{2}$ Department of Elderly, Mondriaan Mental Health Care, Heerlen, Netherlands

${ }^{3}$ Alzheimer Center Limburg, School of Mental Health \& Neurosciences, Department of Psychiatry and Psychology, Maastricht University Medical Centre, Maastricht, Netherlands

${ }^{4}$ Faculty of Health, Medicine and Life Sciences (FHML), Care and Public Health Research Institute (CAPHRI), Department of Methodology and Statistics, Maastricht University, Maastricht, Netherlands

Correspondence

Janine Collet, Department of Elderly, Mondriaan Mental Health Care, Heerlen, The Netherlands.

Email:j.collet@mondriaan.eu

\section{Funding information}

CZ-Health Insurance Company [award number 20120010.]
\end{abstract}

\section{Accessible summary}

What is already known about the subject?

- Working in long-term care is seen as a stressful, physically and mentally demanding occupation, and thus, nursing staff are at risk for work and stress-related diseases.

- In older patients, psychiatric illnesses often occur in combination with physical illnesses, requiring nursing care that is specific to these combined care needs. The impact of caring for these patients on the mental well-being of nurses is unknown.

What this paper adds to existing knowledge?

- Nursing staff working on specialized units for patients with combined care needs experience high levels of self-efficacy in combination with strong feelings of selfrated competence.

- Although levels of burnout are relatively low, mental healthcare nursing staff is more at risk for burnout when working in specialized settings for patients with combined care needs than nursing home staff working in specialized settings for these patients.

- Nursing staff characteristics, such as years of working experience and age, seem more important in relation to staff well-being than patient characteristics in specialized settings for combined care needs.

What are the implications for practice?

- Staff well-being might benefit from specializing care, so that patients with similar care needs are placed together and care is focused.

- The presence of specialized care units for older patients with combined care needs can allow for both targeted and focused allocation of nursing staff to these units and provision of specific training.

\section{Abstract}

Introduction: In older patients, psychiatric illnesses frequently exist in tandem with physical illnesses, requiring nursing care that is specific to these combined care needs. The impact of caring for these patients on the mental well-being of nursing staff is unknown.

Aim: To investigate whether care characteristics of patients with combined care needs are related to the mental well-being of nursing staff. 
Method: Well-being of nursing staff was studied within a larger exploratory observational cross-sectional study that examined the differences and similarities of specialized combined care units in Dutch mental healthcare and nursing home settings.

Results: Nursing staff across settings, with more than 5 years of work experience, felt competent in caring for patients with combined care needs. No significant effects of care characteristics of patients with combined care needs on the work-related wellbeing of nursing staff were shown. Both mental health nursing staff and older employees, however, were found to be more at risk for burnout.

Implications/Conclusion: Staff well-being might benefit from placing patients with combined care needs together, so care is focused. The presence of specialized care units can allow for both targeted and focused allocation of nursing staff to these units and provision of specific training.

\section{KEYWORDS}

mental health care, nursing home care, nursing staff, patients with combined care needs, work-related well-being

\section{1 | INTRODUCTION}

A large percentage of older adults have multiple morbidities and have combined mental (psychiatric and/or cognitive) and physical problems (Fullerton, McGuire, Feng, Mor, \& Grabowski, 2009; Grabowski, Aschbrenner, Rome, \& Bartels, 2010; Health Council of the Netherlands, 2008). These older patients with combined care needs require a combination of physical, psychological and/or psychiatric care (Bartels, 2004; Snowdon, 2010).

Traditionally, nursing homes provide long-term care (LTC) for persons with either cognitive or physical disabilities, and mental healthcare institutions provide LTC for persons with chronic mental illnesses. Earlier studies have shown that older patients with combined care needs benefit from collaborative psychiatric and physical medicine approaches, such as from multidisciplinary care teams that deliver integrated mental and physical care (Bartels, 2004; Collet, de Vugt, Verhey, \& Schols, 2010; Inventor et al., 2005).

Research in these combined care settings for patients with combined care needs is scarce and only consists of describing demographic characteristics, physical morbidity and psychiatric morbidity of this specific patient group. Results from these studies show that patients with combined care needs tend to be younger, more often male, unmarried, have severe challenging behaviours, a high amount of physical comorbidities, high care dependency and cognitive impairment (Collet, de Vugt, Verhey, Engelen, \& Schols, 2016; Seitz, Purandare, \& Conn, 2010; Van den Brink, Gerritsen, de Valk, Oude Voshaar, \& Koopmans, 2017).

Professional caregivers in combined care settings will be challenged by these care complexities, as they must be able to address both somatic care needs and psychiatric and psychogeriatric care needs (Collet et al., 2016; Van den Brink et al., 2017). Several studies looking at combined care needs in general settings confirm this challenge of skills. Blythe et al. stated that mental healthcare nursing staff often lack enough training to meet the physical care demands of patients with combined care needs (Blythe \& White, 2012). According to Beck et al., nurse assistants are primarily educated to provide physical care, and Gabrowski et al. found evidence for nursing home nurses to be under qualified to provide psychiatric care for elderly people (Beck, Doan, \& Cody, 2002; Grabowski et al., 2010). Nursing home nurses themselves have expressed a need for more training in psychiatric care to be able to meet the needs of patients with combined care needs (Dorland, 2007; Molinari et al., 2008).

This challenge of skills could have implications for nursing staff's experienced work-related stress and may therefore have an impact on their well-being and risk for burnout. Staff burnout has been associated with a higher tendency to leave the nursing profession (Leiter \& Maslach, 2009; Simon, Müller, \& Hasselhorn, 2010). Insight into factors influencing the work-related well-being of nursing staff for patients with combined care needs is therefore essential to create both a healthy work environment and a sustainable workforce related to care for this specific group.

Previous studies in general settings showed that patient characteristics can influence work-related well-being of nursing staff and that results are not consequently pointing in one direction.

Residents' challenging behaviour was found to be a stressor for nursing home nurses, and it had an impact on their general health, job satisfaction and work ability (Schmidt, Dichter, Palm, \& Hasselhorn, 2012; Zwijsen et al., 2015). A study by Testad et al., however, showed that agitation and dementia severity are not related to carer stress (Testad, Mikkelsen, Ballard, \& Aarsland, 2010).

In addition, nursing staff characteristics, such as workload, work experience and educational level, have been identified as factors to cause job stress (Hannan, Norman, \& Redfern, 2001; Maslach, Schaufeli, \& Leiter, 2001), while lack of adequate staffing was the 
most frequently mentioned stressor in a study on job burnout comparing psychiatric hospital and community nurses (McTiernan \& McDonald, 2015). A recent meta-analysis of the influence of age on the three dimensions of burnout syndrome (emotional exhaustion, depersonalization and personal accomplishment) in nurses indicated that younger age was a significant factor in the emotional exhaustion and depersonalization of nurses, although it was somewhat less influential in the dimension of personal accomplishment (Gómez-Urquiza José, 2017). Wynd found that registered nurses who scored the highest on levels of professionalism had many years of experience in nursing practice and higher educational degrees (Wynd, 2003). Mental healthcare nursing staff with an average of 13 years of work experience reported positive attitudes towards their role in the physical care for psychiatric patients; they felt more confident about both performing routine physical health observations and their technical skills (Robson, Haddad, Gray, \& Gournay, 2013). In a multilevel study of general hospital nurses, nurse self-efficacy on an individual level was the strongest predictor of the burnout dimensions exhaustion and depersonalization (Consiglio, Borgogni, Vecchione, \& Maslach, 2014). Queiros et al. found that mental distance is influenced by age, gender and job satisfaction and that personal accomplishment is influenced by work experience (Queiros, Carlotto, Kaiseler, Dias, \& Pereira, 2013). Perceived competence in providing dementia care has been associated with increased job satisfaction (Zimmerman et al., 2005; Zwijsen et al., 2015).

Environmental characteristics, such as small-scale living facilities with more personalized care, were reported to reduce care staff stress (De Rooij, Luijkx, Declercq, Emmerink, \& Schols, 2012 Te Boekhorst, Willemse, Depla, Eefsting, \& Pot, 2008; Zimmerman et al., 2005). Pekkarinen et al. concluded that work stressors might be reduced by specializing care, so that residents with similar care needs are placed together and care is focused (Pekkarinen et al., 2006). This is important for staff and patient well-being as nurses working in an environment with lower mental distress hold a less negative attitude towards patients, and their personal accomplishment is higher (Van Bogaert, Dilles, Wouters, \& Van Rompaey, 2014; Wang, Liu, \& Wang, 2015)

\section{2 | AIM}

The primary aim of this study was to explore the work-related wellbeing of nursing staff in relation to patient characteristics in specialized units for older patients with combined care needs in both the nursing home setting and the mental healthcare setting. The study addresses the following research question: what are the personal characteristics (educational level, work experience, age and gender) and the work-related well-being characteristics (mental problems, job satisfaction, perceived self-efficacy and burnout) of the nursing staff caring for older patients with combined care demands?

Because of the traditional categorization in physical care and psychiatric care, and therefore a better training level of nursing staff related to only one of these care domains, we hypothesized that care dependency, physical care demands and intensity of neuropsychiatric symptoms in patients with combined care needs will be correlated to higher symptoms of job burnout and work-related distress in nursing staff across settings. Consistent with results from earlier studies in different settings, we expected higher educated, more experienced and highly (self-rated) competent nursing staff to show fewer symptoms of burnout and work-related mental distress. Data on these specific nurse characteristics were therefore also collected.

The findings of this study are relevant for planning of services and training to maximize well-being of staff and patients in specialized units for combined care needs, taking into account both the possible differential impact of care characteristics of these patients and staff characteristics in different specialized care settings.

\section{3 | METHOD}

\section{1 | Design}

This study was part of a large exploratory observational crosssectional study on the "Specific Care in the Interface of Mental health and Nursing homes" (SpeCIMeN). Patients and nursing staff from two types of care settings for patients with combined care needs were included: the mental healthcare setting and the nursing home setting. Data were collected from November 2013 through April 2015.

The first part of the SpeCIMeN study investigated both the personal and care characteristics and the perceived quality of care of patients with combined care needs residing in specialized care units in both settings. The results of the first part of the SpeCIMeN study have been published elsewhere (Collet et al., 2016).

This study explores the work-related well-being of nursing staff working in specialized units for patients with combined care needs across the nursing home and the mental healthcare setting, related to the care characteristics of these patients and nursing staffs' personal characteristics.

\section{2 | Participants}

Participants (both patients and nursing staff) were recruited from nursing homes and mental healthcare institutions with specialized units for older patients with combined care needs, situated in the southernmost part of Limburg, a province of the Netherlands. To be included, these specialized combined care units needed to fulfil the criteria of delivering integrated multidisciplinary care for older patients with care needs on the combination of psychiatric, cognitive and physical care problems.

Four specialized units within a mental healthcare institution and seven specialized units within two nursing home organizations were identified and included in the study, with a total inclusion of 80 patients with combined care needs in a mental healthcare setting, and 83 nursing home patients, respectively. The specialized units within the mental healthcare setting varied from 14 to 20 persons, while these units within the nursing homes varied from 8 to 20 persons. 
Patients were included if: (1) they stayed for at least 6 weeks on the specialized unit; (2) they had a clear combination of psychiatric, cognitive and physical care problems; and (3) informed consent to participate in the study was given either personally or by an officially appointed legal representative. Patients were excluded if they: (1) refused to participate; and (2) were temporarily admitted to the specialized unit because of admission problems elsewhere.

All nursing staff with permanent employment contracts, working on specialized units for patients with combined care needs in either a nursing home $(n=96)$ or a mental healthcare setting $(n=53)$, were invited to participate in this study. They were included if they: (1) gave informed consent to participate in the study; and (2) worked in direct care with patients with combined care needs. Nurses were excluded if they: (1) had a temporarily employment contract; (2) refused to participate in the study; or (3) were absent because of sickness, during a period of more than 6 weeks.

\section{3 | Procedure}

\subsection{1 | Patients}

We asked the unit physician (psychiatrist or elderly care physician) to identify and inform all patients able to participate in our study. These patients, their representatives and their professional caretakers received an information letter giving details about the nature of the study, the purposes, duration and possible consequences in a form they could understand. If needed, the information was given orally. We further explained that confidentiality was ensured and that permission might be revoked at any time by the patient or the legal representative without further consequences. We then asked the patient or the legal representative to sign the informed consent. All interviews were conducted by a certified elderly care physician (researcher JC) or a trained research assistant. If we sensed any distress in participants during the interview, we offered them the opportunity to return at a more favourable time, skip questions or discontinue participation. Medical files were intensively studied by the researcher (JC), to collect information on psychiatric and somatic morbidity. Vocational nurses, who were the primary responsible caretakers of the patients with combined care needs, provided data on care dependency and current behaviour.

\subsection{2 | Nursing staff}

All nursing staff with permanent employment contracts working on specialized combined care units within a mental healthcare setting $(n=53)$ or a nursing home $(n=96)$ received a letter explaining the study and an informed consent form at their work address. After obtaining informed consent from the nursing staff, a paper questionnaire was given to them at their work address. Approximately 20 min was needed to complete the questionnaire. The questionnaire contained questions on basic personal characteristics, work satisfaction, feelings of competence, work-related mental health problems and burnout. In case of nonresponse after 8 weeks, a reminder was sent by mail.

\section{4 | Measurements}

\subsection{1 | Nursing staff characteristics}

Personal characteristics, such as gender, age, level of professional education (low = low vocational training [nursing assistants], medium = medium vocational training [certified vocational nurses] and high $=$ high vocational training [bachelor degree nurses]) and level of work experience ( $<5$ years or $\geq 5$ years), were retrieved from the selfreport questionnaire.

Work satisfaction was measured by asking professionals to grade their work satisfaction on a scale from 1 to 10 . A higher score expresses more work satisfaction.

Self-rated competence was measured using the Dutch version of the General Self-Efficacy Scale (Schwarzer, Jerusalem, \& Teeuw, 1994). This validated and reliable questionnaire (Cronbach's alpha $=0.80$ ) measures the style of problem solving related to the nursing job and consists of 10 statements that must be answered on a 4-point Likert scale. Answers could vary from 1 (not at all relevant) to 4 (very relevant), meaning that total self-efficacy score varies between 10 and 40. A higher sum score indicates a better style of problem solving and therefore a higher level of self-rated competence. Additionally, professional caregivers were also asked to grade their experienced level of competence in problem solving on a scale of 1-10. A higher score correlates with a greater feeling of competence.

Mental distress such as anxiety, depression and social withdrawal was measured using the General Health Questionnaire (GHQ-12). The 12 -item version is reliable for nursing staff (Cronbach's alpha $=0.76$ ). It has a 4-point response scale, varying from 0 (not at all) to 3 (much more than usual). A higher sum score corresponds with a higher amount of work-related mental health problems (Goldberg et al., 1997).

Burnout was measured using the Utrecht Burnout Scale (UBOS), which is the Dutch version of the Maslach Burnout Inventory (Maslach \& Jackson, 1986; Schaufeli \& van Dierendonck, 2000). We used the UBOS-C, because this version specifically measures the work-related psychological state of individuals who have an occupation with frequent client interaction. UBOS-C provides a reliable (Cronbach's alpha $=0.79$ ) and valid measure of experienced pressure at work. The results can be used for both individual assessment and for comparison of groups. UBOS-C consists of 20 items that can be answered on a 7-point scale, ranging from 0 (never) to 6 (every day). Three following subscales are available: (1) emotional exhaustion measures feelings of being emotionally overextended and exhausted by one's work (eight items; a higher score means more exhaustion); (2) depersonalization assesses impersonal responses towards clients (five items; a higher score means more depersonalization); and (3) personal accomplishment examines feelings of competence and achievement in work (seven items; a higher score means more personal accomplishment). Means of emotional exhaustion, depersonalization and personal accomplishment were calculated with eight, five and seven items, respectively (Schaufeli \& van Dierendonck, 2000). Responses for each subscale were categorized as low, moderate or high in accordance with normative data derived from a sample of 1907 mental 
TABLE 1 UBOS normative data $\left(n=1,907^{\mathrm{a}}\right)$

\begin{tabular}{llll}
$\begin{array}{l}\text { UBOS subscales } \\
\text { (range 0-6) }\end{array}$ & Low & Moderate & High \\
\hline Emotional exhaustion & $0.39-1.12$ & $1.13-2.49$ & $2.50-3.98$ \\
\hline Depersonalization & $0.01-0.59$ & $0.60-1.79$ & $1.80-3.18$ \\
\hline Accomplishment & $3.30-4.13$ & $4.14-5.42$ & $5.43-5.98$ \\
\hline
\end{tabular}

${ }^{a}$ Categorization of low, average and high burnout according to normative data. This sample included mental health nursing staff and nursing home nursing staff (Schaufeli \& van Dierendonck, 2000).

health professionals in LTC facilities. These normative cut-points are applied for both the nursing home and the mental health settings (See Table 1).

\subsection{2 | Patient characteristics-Data collected from medical files}

Baseline characteristics, such as age, sex, time of institutionalization and level of education, were retrieved from the patient's record.

Current physical disorders were collected from data on the medical diagnosis, the available laboratory results and the medication use, as stated within the patients' medical record.

Severity of all physical disorders was scored using the 14-item version of the Cumulative Illness Rating Scale (CIRS) 14-item version (Miller et al., 1992). This scale measures multimorbidity in the light of all medical problems encountered in a geriatric population. The theoretical scores range from 0 to 56 based on scoring the severity of co-occurring medical conditions from 0 (no problem) to 4 (extremely severe problem). In this study, the severity of co-occurring medical conditions was scored excluding the psychiatry CIRS subscale.

Psychiatric illnesses were classified according to the Diagnostic and Statistical Manual of Mental Disorders. All DSM classifications made by a psychiatrist or trained psychologist and stated in the patients' medical record were accepted. Both axis I and axis II classifications were noted (DSM-IV-TR. Vol. 4th edition, Text Revision. 2000).

\subsection{3 | Patient characteristics-Data provided by vocational nurses}

Patients' needs and care dependency were measured using the Care Dependency Scale (CDS) (Dijkstra, Buist, \& Dassen, 1998; Dijkstra, Tiesinga, Plantinga, Veltman, \& Dassen, 2005). CDS was shown to have excellent reliability for several countries (Cronbach's alpha $=0.95-0.97$ ), and it can be used for assessment purposes, both at the group and individual levels (Polit, Beck, \& Hungler, 2004). The CDS measures a patients' ability to perform activities independently. It consists of 15 categories that are scored using a 5-point Likert-type scale. Responses range from 1 to 5 , where 1 is completely dependent and 5 is almost independent. Patients with a total CDS score of $\leq 68$, are classified as care-dependent.

Neuropsychiatric symptoms (NPS) were assessed using the Dutch version of the Neuropsychiatric Inventory (NPI) (Cummings et al.
1994; Kat et al., 2002). Content validity of the NPI is high which was rated by a panel of 10 experts in geriatric psychiatry. Inter-rater reliability ranged from $93.6 \%$ to $100 \%$, depending on the subdomain. Test-retest reliability was high, with a Pearson correlation of 0.79 (Cummings, 1997). The NPI includes 12 neuropsychiatric symptoms. The frequency and severity of each symptom are rated on a 5-point (0-4) and 4-point (0-3) Likert scale, respectively. The total score equals the product of frequency and severity scores. NPI symptoms were considered clinically relevant when the total scores were $\geq 4$.

\subsection{Ethical considerations}

The local Medical Ethics Committee of the Maastricht University Medical Centre approved the study (number 134049) after reviewing the study protocol, and considered it to be not subject to the Medical Research Involving Human Subjects Act. The study was also approved by the Board of Directors and the Client Advisory councils of all the participating institutions. Patients' and nursing staff participation was voluntary, and data collection was conducted confidentially and anonymously. Informed consent to participate in the study was given either personally or by the patient's officially appointed legal representative.

\section{6 | Statistical analyses}

Categorical variables are presented as the number (\%), while numerical variables are presented as the mean (standard deviation [SD]) or median (interquartile range (IQR), i.e., 25th-75th percentile), where appropriate. Comparisons between nursing staff groups (mental healthcare setting versus nursing home setting) were performed using chi-square tests for nominal or ordinal variables, and independentsamples $t$ tests or Mann-Whitney $U$ tests where appropriate for numerical variables.

Four analyses were performed using a linear mixed model (LMM) in which a random intercept for the specialized unit was used to account for the correlation between professionals from the same specialized unit. The first analysis examined the effect of the independent variable setting, care dependency, physical comorbidity and neuropsychiatric symptoms of residents with complex care needs on the dependent variable GHQ-12 score (work-related mental health problems), whereas the other three analyses examined these effects on the dependent variable UBOS-C subscales (emotional exhaustion, depersonalization and personal accomplishment). In all four analyses, types of setting were combined ( $n=100$ for combined settings), and all independent variables (either numerical or binary) were included simultaneously into the model.

In addition, relevant background variables such as gender, age, educational level (categorical: low or medium/high) and work experience ( $<5$ or $\geq 5$ years) were also included in these analyses. Because of the exploratory nature of the study, no adjustment for multiple testing was made, and we focused on the size of the observed effects. Multicollinearity was tested using variance inflation factors (VIFs). IBM SPSS Statistics for Windows (version 21.0) was 
used for the statistical analyses. A $p$-value $\leq .05$ was considered statistically significant.

\section{4 | RESULTS}

The questionnaire was completed by 37 of the 53 professionals with a permanent employment contract, working within a mental healthcare specialized unit (response rate, $70 \%$ ), and by 73 of the 96 professionals with a permanent employment contract, working in a nursing home specialized unit (response rate, 76\%). Reasons for nonresponse were absence because of illness or lack of interest in participating.

\subsection{Demographic characteristics of nursing staff}

The basic characteristics of the nursing staff are presented in Table 2 .

Except for the educational level $(p<.001)$, there was no significant difference between groups in terms of demographic characteristics. Nursing staff in both settings consisted predominantly of females aged $40-49$ years. The majority of the staff had more than 5 years of work experience in caring for patients with combined care needs.

\subsection{Work-related mental health characteristics of nursing staff}

The mean scores on GHQ-12, self-efficacy and all subscales of UBOS-C, and grading of competence in problem solving and job satisfaction are presented in Table 3. GHQ-12 scores indicated moderate levels of mental distress in nursing staff in both types of settings. Both groups of nursing staff showed similar strong feelings of competence

TABLE 2 Basic characteristics of nursing staff in mental healthcare and nursing home settings

\begin{tabular}{|c|c|c|c|}
\hline Characteristics & $\begin{array}{l}\text { All nurses } \\
(n=110)\end{array}$ & $\begin{array}{l}\text { MHC nurses } \\
(n=37)\end{array}$ & $\begin{array}{l}\text { NH nurses } \\
(n=73)\end{array}$ \\
\hline $\begin{array}{l}\text { Age (years), Mean } \\
\text { (SD) }\end{array}$ & $42.4(10.7)$ & $40.1(10.5)$ & $43.2(10.8)$ \\
\hline Female, $n(\%)$ & $95.0(86.4)$ & $34.0(91.9)$ & $61.0(83.6)$ \\
\hline \multicolumn{4}{|c|}{ Work experience in specialized unit, $n$ (\%) } \\
\hline$<5$ years & $11.0(10.0)$ & $3.0(8.1)$ & $8.0(11.0)$ \\
\hline$\geq 5$ years & $99.0(90.0)$ & $34.0(91.9)$ & $65.0(89.0)$ \\
\hline $\begin{array}{l}\text { Working hours, } \\
\text { Mean (SD) }\end{array}$ & $28.9(5.8)$ & $28.2(6.3)$ & $29.3(5.5)$ \\
\hline \multicolumn{4}{|c|}{ Level of education, $n$ (\%) } \\
\hline Low & $26.0(23.6)$ & $6.0(13.5)$ & $20.0(28.8)$ \\
\hline Medium & $62.0(56.4)$ & $15.0(40.5)$ & $47.0(64.4)$ \\
\hline High & $21.0(19.1)$ & $16.0(43.2)$ & $5.0(6.8)$ \\
\hline
\end{tabular}

$\mathrm{MHCl}$, mental healthcare setting; $\mathrm{NH}$, nursing home setting; Low, low vocational training (nursing assistants); Medium, medium vocational training (certified vocational nurses); High, high vocational training (bachelor degree nurses). in problem solving. For the UBOS-C, mean overall scores on the emotional exhaustion scale indicated low levels of emotional strain for both groups of nursing staff. Mean overall depersonalization scores indicated low levels of mental distance, with a significant higher score (moderate level) for the mental healthcare nursing staff. Mean overall accomplishment scores showed a moderate level of personal accomplishment for both groups, with a significantly higher score for nursing home nurses. Nursing home nurses also showed a significantly higher level of job satisfaction.

\section{3 | Characteristics of patients with combined care needs}

Patients were predominantly males aged $60-69$ years. The majority of them were institutionalized for more than 1 year. One-third of the patients in mental health care had a psychotic disorder, and in $40 \%$ of those patients, multiple-axis one diagnoses were present. Cognitive disorders, mainly frontotemporal lobe dementia, and alcohol-related dementia were reported in three-quarters of the patients in nursing homes. Personality disorders were diagnosed in one-quarter of all patients. Overall, patients had seven comorbid conditions, and they all showed a nursing care dependency. Patients in a mental healthcare setting had more neuropsychiatric symptoms, with a higher prevalence of delusions, hallucinations and anxiety. Table 4 shows the relevant behavioural and care characteristics as collected within the SpeCIMeN study.

\subsection{Variables associated with work-related well-being in nursing staff}

Table 5 shows the estimated effects that were obtained from linear mixed model (LMM) analyses.

Although effects of characteristics of patients with combined care needs (NPI, CDS, CIRS) on job burnout or work-related mental distress of nursing staff were not significant, the LMM analyses showed that for both groups of nursing staff, a higher amount of neuropsychiatric symptoms was associated with higher rates of both emotional exhaustion (1 point higher NPI score results in 0.02 point higher emotional exhaustion) and work-related mental distress (1 point higher NPI score results in 0.11 point higher GHQ score). It also showed that a higher amount of physical illness severity in patients was associated with both lower emotional exhaustion rates (1 point higher CIRS score results in 0.07 point lower UBOS emotional exhaustion score) and work-related mental distress ( 1 point higher CIRS score results in 0.37 point lower GHQ score).

Within the LMM analyses, several effects were found for both basic personal characteristics of nursing staff and type of setting. A lower level of education was significantly associated with lower rates of depersonalization and lower rates of personal accomplishment. Older professionals had significantly higher rates of emotional exhaustion and showed a tendency towards higher feelings of depersonalization and lower rates of personal accomplishment. For setting, significantly higher rates of depersonalization and lower rates of 
TABLE 3 Competence, mental health and job burnout of nursing staff in mental healthcare and nursing home setting

\begin{tabular}{|c|c|c|c|c|c|c|}
\hline Measurement & $\begin{array}{l}\text { All nurses }(n=110) \\
\text { Mdn (IQR) }\end{array}$ & $\begin{array}{l}\text { MHC }(n=37) \\
M d n(I Q R)\end{array}$ & $\begin{array}{l}\mathrm{NH}(n=73) \\
\mathrm{Mdn}(\mathrm{IQR})\end{array}$ & $U^{\mathrm{a}}$ & $Z^{a}$ & $p^{\mathrm{a}}$ \\
\hline GHQ-12 $2^{b}$ & $9.00(6.00)$ & $10.00(7.00)$ & $9.00(5.00)$ & 1141.00 & -1.33 & .183 \\
\hline $\begin{array}{l}\text { UBOS }^{\mathrm{C}} \text { emotional } \\
\text { exhaustion }\end{array}$ & $1.00(0.88)$ & $1.13(0.81)$ & $0.88(1.16)$ & 1086.50 & -1.68 & .094 \\
\hline Grade of competence $^{d}$ & $8.00(1.00)$ & $8.00(0.00)$ & $8.00(1.00)$ & 1173.00 & -1.10 & .270 \\
\hline Grade of job satisfaction $^{d}$ & $8.00(1.00)$ & 7.00 (1.00) & $8.00(0.00)$ & 905.00 & -3.03 & .002 \\
\hline
\end{tabular}

Mdn, median; IQR, interquartile range $\left(\mathrm{P}_{25}-\mathrm{P}_{75}\right)$; $\mathrm{MHC}$, mental healthcare setting; $\mathrm{NH}$, nursing home setting.

${ }^{\mathrm{a}} \mathrm{Mann}-$ Whitney $U$ test: significance level is 0.05 .

${ }^{\mathrm{b}}$ General Health Questionnaire, twelve items.

'Utrecht Burnout Scale: range 0-6: norms see Table 1.

${ }^{\mathrm{d}}$ Grade on a scale from 0 to 10 .

\begin{tabular}{|lcccc|}
\hline & $\begin{array}{l}\text { All CCN } \\
(n=163) \\
\text { Mean (SD) }\end{array}$ & $\begin{array}{l}\text { MHC ( }=80) \\
\text { Mean (SD) }\end{array}$ & $\begin{array}{l}\text { NH }(n=83) \\
\text { Mean (SD) }\end{array}$ & $p^{\text {a }}$ \\
\hline Multimorbidity (count) & $7.0(2.2)$ & $7.1(2.1)$ & $6.9(2.3)$ & .945 \\
\hline Care Dependency Scale & $45.0(15.5)$ & $43.9(13.8)$ & $46.1(17.1)$ & .376 \\
\hline Cumulative Illness Rating Score & $15.2(5.0)$ & $15.4(4.7)$ & $14.9(5.3)$ & .969 \\
\hline $\begin{array}{l}\text { Neuropsychiatric Inventory } \\
\text { (total) }\end{array}$ & $29.5(21.1)$ & $32.6(19.5)$ & $25.9(22.5)$ & .009 \\
\hline
\end{tabular}

TABLE 4 Behavioural and care characteristics of patients with combined care needs in mental healthcare and nursing home settings

$\mathrm{CCN}$, patients with combined care needs; $\mathrm{MHC}$, mental healthcare setting; $\mathrm{NH}$, nursing home setting. ${ }^{a}$ chi-square, significance level is 0.05 .

personal accomplishment were found for mental healthcare nursing staff.

\section{5 | DISCUSSION}

To the best of our knowledge, this is the first study to report and compare data on the mental well-being of nursing staff working on specialized units for patients with combined care needs across the nursing home and the mental healthcare setting and the possible relationship with the care characteristics of these patients.

The findings show that, although neither group showed high levels of burnout, mental health nurses, working on specialized units for patients with combined care needs, clearly experience more mental distress than nursing home nurses. Mental healthcare nursing staff showed significantly higher depersonalization, lower personal accomplishment and a lower level of job satisfaction, and were therefore more at risk for burnout. Compared to nursing home nurses, mental healthcare nursing staff thus seems to respond less personally towards patients with combined care needs, and they also experience a significantly lower sense of achievement when interacting/working with these patients.
Possible explanations for these differences between settings could be the differences in patient characteristics, such as the psychiatric multimorbidity in mental healthcare patients, or the differences in workplace characteristics, such as the organizational position of the specialized care unit or the nursing staffs' experienced appreciation within the organization (Van Bogaert et al., 2014; Wang et al., 2015). But contrary to what we expected, we did not find a relationship between patient characteristics and staff well-being across both settings. Based on our previous study, we expected that due to a higher prevalence of neuropsychiatric symptoms in mental healthcare patients with combined care needs, well-being of nursing staff would be more compromised in this setting as they have to encounter higher instances of challenging behaviours. The fact that we did not find a difference between both settings might suggest that within both settings, a fair allotment of personnel to specialized units already exists, meaning that current staff is equally capable of dealing with both clinical diseases and neuropsychiatric symptoms.

We did find a tendency that for both groups of nursing staff, a higher amount of NPS in persons with combined care needs is associated with higher rates of both emotional exhaustion and work-related mental distress. This finding is consistent with studies in dementia care settings (Schmidt et al., 2012; Zwijsen et al., 2015). Results also 
TABLE 5 Effect of NPI, CDS, CIRS, basic characteristics of nursing staff and type of setting on GHQ-12

\begin{tabular}{|c|c|c|c|c|c|}
\hline \multirow[b]{2}{*}{ Outcome variable } & \multirow[b]{2}{*}{ Explorative variables* } & \multirow{2}{*}{$\begin{array}{l}\text { Estimated } \\
\text { effect (B) }\end{array}$} & \multicolumn{2}{|l|}{$95 \% \mathrm{Cl}$} & \multirow[b]{2}{*}{$p$} \\
\hline & & & Lower & Upper & \\
\hline \multirow[t]{8}{*}{ GHQ-12 } & NPI & 0.11 & -0.00 & 0.23 & .057 \\
\hline & CDS & -0.00 & -0.13 & 0.13 & .992 \\
\hline & CIRS & -0.37 & -0.77 & 0.03 & .068 \\
\hline & $\begin{array}{l}\text { Education (low/ } \\
\text { medium and high) }\end{array}$ & -2.1 & -5.0 & 0.78 & .150 \\
\hline & $\begin{array}{l}\text { Work experience } \\
\text { (<5 years } / \geq 5 \text { years })\end{array}$ & -3.1 & -6.8 & 0.65 & .105 \\
\hline & Gender (male/female) & -2.4 & -5.4 & 0.65 & .122 \\
\hline & Age & -0.01 & -0.11 & 0.10 & .886 \\
\hline & Setting $(\mathrm{MHC} / \mathrm{NH})$ & -0.11 & -2.7 & 2.5 & .936 \\
\hline \multirow{8}{*}{$\begin{array}{l}\text { UBOS - emotional } \\
\text { exhaustion }\end{array}$} & NPI & 0.02 & -0.00 & 0.04 & .089 \\
\hline & CDS & -0.01 & -0.04 & 0.02 & .256 \\
\hline & CIRS & -0.07 & -0.16 & 0.02 & .089 \\
\hline & $\begin{array}{l}\text { Education(low/ } \\
\text { medium \&high) }\end{array}$ & -0.30 & -0.71 & 0.11 & .145 \\
\hline & $\begin{array}{l}\text { Work experience } \\
\text { (<5 years } / \geq 5 \text { years })\end{array}$ & 0.01 & -0.51 & 0.54 & .958 \\
\hline & Gender(male/female) & 0.04 & -38 & 0.46 & .856 \\
\hline & Age & 0.02 & 0.00 & 0.03 & .028 \\
\hline & Setting (MHC/NH) & 0.01 & -0.49 & 0.52 & .953 \\
\hline \multirow[t]{8}{*}{ UBOS depersonalization } & NPI & 0.01 & -0.01 & 0.02 & .311 \\
\hline & CDS & -0.01 & -0.02 & 0.01 & .486 \\
\hline & CIRS & -0.03 & -0.07 & 0.01 & .184 \\
\hline & $\begin{array}{l}\text { Education(low/ } \\
\text { medium and high) }\end{array}$ & -0.34 & -0.64 & -0.03 & .030 \\
\hline & $\begin{array}{l}\text { Work experience } \\
\text { (<5 years } / \geq 5 \text { years })\end{array}$ & 0.02 & -0.37 & 0.40 & .939 \\
\hline & Gender(male/female) & -0.10 & -0.42 & 0.22 & .536 \\
\hline & Age & 0.01 & -0.00 & 0.02 & .066 \\
\hline & Setting (MHC/NH) & 0.39 & 0.11 & 0.66 & .006 \\
\hline \multirow[t]{8}{*}{ UBOS accomplishment } & NPI & -0.01 & -0.04 & 0.02 & .492 \\
\hline & CDS & 0.02 & -0.02 & 0.05 & .207 \\
\hline & CIRS & 0.05 & -0.06 & 0.16 & .282 \\
\hline & $\begin{array}{l}\text { Education (low/ } \\
\text { medium \&high) }\end{array}$ & -0.60 & -1.0 & -0.17 & .007 \\
\hline & $\begin{array}{l}\text { Work experience } \\
(<5 \text { years } / \geq 5 \text { years })\end{array}$ & -0.29 & -0.85 & 0.27 & .308 \\
\hline & Gender (male/female) & 0.51 & 0.06 & 0.96 & .027 \\
\hline & Age & -0.02 & -0.03 & 0.00 & .055 \\
\hline & Setting (MHC/NH) & -0.92 & -1.6 & -0.27 & .012 \\
\hline
\end{tabular}

$95 \% \mathrm{Cl}, 95 \%$ confidence interval; $p$, significance level is 0.05; UBOS, Utrecht Burnout Scale; GHQ-12, General Health Questionnaire 12 items; NPI, Neuropsychiatric Inventory (measures neuropsychiatric symptoms); CDS, Care Dependency Scale (measures ADL dependency); CIRS, Cumulative Illness Rating Scale (measures severity of physical comorbidity); Education, low vocational training (nursing assistant) versus medium vocational training (registered vocational nurse) and high vocational training (bachelor degree nurse); $\mathrm{MHC}$, mental healthcare setting; $\mathrm{NH}$, nursing home setting.

*Mean difference: NPI, CDS and CIRS per unit increase, Age per year. Multicollinearity diagnostics showed no collinearity problem (all VIFs $\leq 1.66$ ). 
showed a tendency towards a higher amount of physical illness severity in persons with combined care needs that is associated with both lower emotional exhaustion rates and work-related mental distress in nursing staff in both settings. This result is consistent with earlier findings showing that work experience has a positive effect on job performance and a more positive attitude towards physical care for patients with a mental illness (Queiros et al., 2013; Robson et al., 2013). Further exploration and confirmation using additional research is needed to be able to attach the correct value to these tendencies found in our study.

Looking into staff demographic characteristics, we found that both the mean age and work experience of staff were similar in both settings. We further found that nursing staff in both settings were mainly female, which is not surprising, because worldwide nursing is still a female-dominated profession. There was a significant difference in the educational levels across settings, with low education levels for $29 \%$ of nursing home nurses. But this did not result in differences in feelings of self-efficacy and levels of mental distress as these were similar in both settings, indicating that educational level alone is not an indicator of work-related mental well-being of nursing staff in these specialized units. Possibly years of work experience are more important in relation to self-efficacy than educational level. In our study, the nursing staff in both settings had more than 5 years of work experience on specialized units, thereby affirming earlier study results, that in gaining more experience, nursing staff in both settings probably became more qualified to take care of patients with combined care needs (Robson et al. 2013; Wynd, 2003). But the educational level was related to other aspects of staff well-being. A lower level of education was significantly associated with lower rates of depersonalization and lower rates of personal accomplishment. Age of the staff also appeared to be relevant, as older professionals had significantly higher rates of emotional exhaustion and showed a tendency towards higher feelings of depersonalization and lower rates of personal accomplishment. This finding is contradictory to the results of a recent study of Gomez-Urquiza, where younger age was a significant factor in emotional exhaustion, personal accomplishment and depersonalization of nurses (GómezUrquiza José, 2017).

\section{W WHAT THIS PAPER ADDS TO EXISTING EVIDENCE}

The present study shows that, across settings, nurses showed a highly perceived self-efficacy in combination with strong feelings of selfrated competence, when working on specialized units for patients with combined care needs. Mental health nurses and older nurses however experience more mental distress in working with this specific group of patients, than nursing home nurses. The study suggests that there is no significant relationship between care characteristics of patients with combined care needs and mental distress in nurses who are working on specialized units for these patients.

Our study findings affirm the findings of Pekkarinen that work stressors may be reduced by placing patients with similar care needs together in specialized LTC-units, so that care can be focused (Pekkarinen et al., 2006). They also affirm the findings of Quiros, Robson and Wynd that nurses with more work experience show both a higher job performance and a more positive attitude towards physical care for patients with mental illness (Queiros et al., 2013; Robson et al., 2013; Wynd, 2003). The results of both the present and earlier studies can therefore serve as building blocks for management to organize focused care for patients with combined care needs on specialized units, with an optimal selection of experienced and educated nursing staff.

\section{7 | STRENGTHS AND LIMITATIONS OF THE STUDY}

This study is a first exploratory step towards gaining deeper insight into the mental distress and burnout of nursing staff, working on specialized units for patients with combined care needs. Although there was a high response rate in both settings, the study may be limited in its power to demonstrate representative characteristics of nursing staff because of the use of a selected cohort of predominantly female nursing staff, without a direct comparison to nursing staff in nonspecialized care units. Because this is a Dutch study, its representativeness of other countries remains unknown. The findings pertaining to the LMM should be treated with caution because the sample size is relatively small $(n=100)$, with a large variance (4-17) in participating nursing staff per unit, and thus, the reliability on unit level and generalizability of the findings are limited. Because we used a general self-efficacy questionnaire and measure of competence, no conclusions can be drawn about the confidence that nursing staff have in providing physical care versus psychiatric care.

\section{8 | IMPLICATIONS FOR RESEARCH AND PRACTICE}

Study results show that nursing staff, working on specialized combined care units across settings, with more than 5 years' work experience, feels competent in caring for patients with combined care needs. They do not experience high feelings of burnout and experience high feelings of self-efficacy and accomplishment. Nevertheless, management should be especially attentive to mental health nursing staff caring for patients with combined care needs. They seem to be more at risk for burnout, as they indicate a lower level of work satisfaction and a higher degree of depersonalization. Older employees in combined care settings also deserve more attentiveness, as they demonstrate lower feelings of accomplishment and a higher degree of depersonalization. To provide in a sustainable workforce, it is important for healthcare organization leaders to focus both on just allotment of personal and on implementing strategies that provide adequate support to nursing staff. Several psychological empowerment programmes for nurses were shown to be successful in improving nurses' physical and mental health 
and quality of work life; improving patient satisfaction; and reducing associated costs of burnout (Addo, Stephen, \& Kirkpatrick, 2012; Henry, 2014; McTiernan \& McDonald, 2015; Nowrouzi et al., 2015). Future studies can be worthwhile to identify nursing staffs' specific needs, e.g., their skills and confidence in providing physical care versus psychiatric care, their coping styles and their perceived facilitators or barriers in the organization of care for older patients with combined care needs to develop and implement a tailored intervention program for this group of nursing staff.

\section{9 | RELEVANCE STATEMENT}

The impact of caring for older patients with combined psychiatric, cognitive and physical care needs on the mental well-being of nursing staff is unknown. We therefore investigated whether care characteristics of patients with combined care needs are related to the mental well-being of nursing staff. Study results show that mental well-being of nursing staff might benefit from placing patients with combined care needs together, so care is focused, and that the presence of specialized care units can allow for both targeted and focused allocation of nursing staff to these units and provision of specific training.

\section{ACKNOWLEDGMENTS}

The authors wish to confirm that the STROBE checklist was followed writing this manuscript. The authors thank Audrey M. M. Janssen, independent psychologist, for her help with data collection and Karin P.M. Aretz, research officer, for her input concerning the analyses. Thanks are also extended to all nursing staff who participated in this research.

\section{CONFLICT OF INTEREST}

There is no potential conflict of interests.

\section{ORCID}

J. Collet iD http://orcid.org/0000-0002-0950-5529

\section{REFERENCES}

Addo, M. A., Stephen, A. I., \& Kirkpatrick, P. (2012). Acute mental health/ psychiatric nurses' experiences of clinical supervision in promoting their well-being in their workplace: A systematic review. JBI Library of Systemic Reviews, 10(56 Suppl), 1-16.

Bartels, S. J. (2004). Caring for the whole person: Integrated health care for older adults with severe mental illness and medical comorbidity. Journal of the American Geriatric Society, 52(12 Suppl), S249-S257. https://doi. org/10.1111/(ISSN)1532-5415

Beck, C., Doan, R., \& Cody, M. (2002). Nursing assistants as providers of mental health care in nursing homes. Generations: the Journal of the Western Gerontological Society, 26(Part 1), 6-71.

Blythe, J., \& White, J. (2012). Role of the mental health nurse towards physical health care in serious mental illness: An integrative review of 10 years of UK Literature. International Journal of Mental Health Nursing, 21(3), 193-201. https://doi. org/10.1111/j.1447-0349.2011.00792.x

Collet, J., de Vugt, M. E., Verhey, F. R. J., Engelen, N. J. J. A., \& Schols, J. M. G. A. (2016). Characteristics of double care demanding patients in a mental health care setting and a nursing home setting: Results from the SpeCIMeN study. Aging and Mental Health, 22, 33-39.

Collet, J., de Vugt, M. E., Verhey, F. R., \& Schols, J. M. (2010). Efficacy of integrated interventions combining psychiatric care and nursing home care for nursing home residents: A review of the literature. International Journal of Geriatric Psychiatry, 25(1), 3-13.

Consiglio, C., Borgogni, L., Vecchione, M., \& Maslach, C. (2014). Selfefficacy, perceptions of context, and burnout: A multilevel study on nurses. La Medicina del Lavoro, 105(4), 255-268.

Cummings, J.L. (1997). The neuropsychiatric inventory: Assessing psychopathology in dementia patients. Neurology, 48, 10-16.

Cummings, J. L., Mega, M., Gray, K., Rosenberg-Thompson, S., Carusi, D. A., \& Gornbein, J. (1994). The neuropsychiatric inventory: Comprehensive assessment of psychopathology in dementia. Neurology, 44(12), 2308-2314. https://doi.org/10.1212/WNL.44.12.2308

De Rooij, A. H. P. M., Luijkx, K. G., Declercq, A. G., Emmerink, P. M. J., \& Schols, J. M. G. A. (2012). Professional caregivers' mental health problems and burnout in small-scale and traditional long term care settings for elderly people with dementia in The Netherlands and Belgium. Journal of the American Medical Directors Association, 13(5), 486. e487486.e411.

Dijkstra, A., Buist, G., \& Dassen, T. (1998). Operationalization of the concept of 'nursing care dependency' for use in long-term care facilities. Australian and New Zealand Journal of Mental Health Nursing, 7(4), 142-151.

Dijkstra, A., Tiesinga, L. J., Plantinga, L., Veltman, G., \& Dassen, T. W. (2005). Diagnostic accuracy of the care dependency scale. Journal of Advanced Nursing, 50(4), 410-416. https://doi. org/10.1111/j.1365-2648.2005.03406.x

Dorland, L. M. (2007). Psychische hulpverlening aan ouderen in verzorgingsen verpleeghuizen. Monitor geestelijke gezondheidszorg Ouderen. Urecht: Trimbos Instituut.

Fullerton, C. A., McGuire, T. G., Feng, Z., Mor, V., \& Grabowski, D. C. (2009). Trends in mental health admissions to nursing homes, 19992005. Psychiatric Services, 60(7), 965-971. https://doi.org/10.1176/ ps.2009.60.7.965

Goldberg, D. P., Gater, R., Sartorius, N., Ustun, T. B., Piccinelli, M., Gureje, O., \& Rutter, C. (1997). The validity of two versions of the GHQ in the WHO study of mental illness in general health care. Psychologica Medica, 27, 191-197. https://doi.org/10.1017/ S0033291796004242

Gómez-Urquiza José, L. J. (2017). Age as a risk factor for burnout syndrome in nursing professionals: A meta-analytic study. Research in Nursing and Health, 40(2), 99-110. https://doi.org/10.1002/nur.21774

Grabowski, D. C., Aschbrenner, K. A., Rome, V. F., \& Bartels, S. J. (2010). Quality of mental health care for nursing home residents: A literature review. Medical Care and Research Review, 67(6), 627-656. https://doi. org/10.1177/1077558710362538

Hannan, S., Norman, I. J., \& Redfern, S. J. (2001). Care work and quality of care for older people: A review of the research literature. Reviews in Clinical Gerontology, 11(2), 189-203.

Health Council of the Netherlands (2008). Health care for the elderly with multimorbidity. The Hague: The Netherlands.

Henry, B. J. (2014). Nursing burnout interventions: What is being done? Clinical Journal of Oncology Nursing, 18(2), 211-214. https://doi. org/10.1188/14.CJON.211-214

Inventor, B. R., Henricks, J., Rodman, L., Imel, J., Holemon, L., \& Hernandez, F. (2005). The impact of medical issues in inpatient geriatric psychiatry. Issues in Mental Health Nursing, 26(1), 23-46. https://doi. org/10.1080/01612840590883591 
Kat, M. G., de Jonghe, J. F., Aalten, P., Kalisvaart, C. J., Droes, R. M., \& Verhey, F. R. (2002). Neuropsychiatric symptoms of dementia: Psychometric aspects of the Dutch Neuropsychiatric Inventory (NPI). Tijdschrift voor Gerontologie en Geriatrie, 33(4), 150-155.

Leiter, M. P., \& Maslach, C. (2009). Nurse turnover: The mediating role of burnout. Journal of Nursing Management, 17(3), 331-339. https://doi. org/10.1111/j.1365-2834.2009.01004.x

Maslach, C., \& Jackson, S. E. (1986). MBI: Maslach burnout inventory: Manual, Research ed. USA: Palo Alto.

Maslach, C., Schaufeli, W. B., \& Leiter, M. P. (2001). Job burnout. Annual Reviews in Psychology, 52, 397-422. https://doi.org/10.1146/annurev. psych.52.1.397

McTiernan, K., \& McDonald, N. (2015). Occupational stressors, burnout and coping strategies between hospital and community psychiatric nurses in a Dublin region. Journal of Psychiatric and Mental Health Nursing, 22(3), 208-218. https://doi.org/10.1111/jpm.12170

Miller, M. D., Paradis, C. F., Houck, P. R., Mazumdar, S., Stack, J. A., Rifai, A. H., ... Reynolds, C. F. (1992). Rating chronic medical illness burden in geropsychiatric practice and research: Application of the Cumulative Illness Rating Scale. Psychiatry Research, 41(3), 237-248. https://doi. org/10.1016/0165-1781(92)90005-N

Molinari, V. A., Merritt, S. S., Mills, W. L., Chiriboga, D. A., Conboy, A., Hyer, K., \& Becker, M. A. (2008). Serious mental illness in Florida nursing homes: Need for training. Gerontology and Geriatric Education, 29(1), 66-83. https://doi.org/10.1080/02701960802074321

Nowrouzi, B., Lightfoot, N., Larivière, M., Carter, L., Rukholm, E., Schinke, R., \& Belanger-Gardner, D. (2015). Occupational stress management and burnout interventions in nursing and their implications for healthy work environments. Workplace Health \& Safety, 63(7), 308-315. https:// doi.org/10.1177/2165079915576931

Pekkarinen, L., Sinervo, T., Elovainio, M., Noro, A., Finne-Soveri, H., \& Leskinen, E. (2006). Resident care needs and work stressors in special care units versus non-specialized long-term care units. Research in Nursing \& Health, 29(5), 465-476. https://doi.org/10.1002/ (ISSN)1098-240X

Polit, D. F., Beck, C. T., \& Hungler, B. P. (2004). Nursing research: Principles and methods. Philadelphia, PA: Lippincott, Williams \& Wilkins.

Queiros, C., Carlotto, M. S., Kaiseler, M., Dias, S., \& Pereira, A. M. (2013). Predictors of burnout among nurses: An interactionist approach. Psicothema, 25(3), 330-335.

Robson, D., Haddad, M., Gray, R., \& Gournay, K. (2013). Mental health nursing and physical health care: A cross-sectional study of nurses' attitudes, practice, and perceived training needs for the physical health care of people with severe mental illness. International Journal of Mental Health Nursing, 22(5), 409-417. https://doi.org/10.1111/j.1447-0349.2012.00883.x

Schaufeli, W., \& van Dierendonck, D. (2000). UBOS Utrecht burnout scale: Manual. The Netherlands; Amsterdam: Harcourt Test Services.

Schmidt, S. G., Dichter, M. N., Palm, R., \& Hasselhorn, H. M. (2012). Distress experienced by nurses in response to the challenging behaviour of residents - evidence from German nursing homes. Journal of Clinical Nursing, 21(21-22), 3134-3142. https://doi.org/10.1111/jocn.12066

Schwarzer, R., Jerusalem, M., \& Teeuw, B.. (1994). General Self-Efficacy Scale, Dutch adaptation. Retrieved from: http://userpage.fu-berlin.de/

Seitz, D., Purandare, N., \& Conn, D. (2010). Prevalence of psychiatric disorders among older adults in long-term care homes: A systematic review. International Psychogeriatrics, 22(7), 1025-1039. https://doi. org/10.1017/S1041610210000608

Simon, M., Müller, B. H., \& Hasselhorn, H. M. (2010). Leaving the organization or the profession - a multilevel analysis of nurses' intentions. Journal of Advanced Nursing, 66(3), 616-626. https://doi. $\operatorname{org} / 10.1111 / \mathrm{j} .1365-2648.2009 .05204 . x$

Snowdon, J. (2010). Mental health service delivery in long-term care homes. International Psychogeriatrics, 22(7), 1063-1071. https://doi. org/10.1017/S1041610210000773

Te Boekhorst, S., Willemse, B., Depla, M. F., Eefsting, J. A., \& Pot, A. M. (2008). Working in group living homes for older people with dementia: The effects on job satisfaction and burnout and the role of job characteristics. International Psychogeriatrics, 20(5), 927-940.

Testad, I., Mikkelsen, A., Ballard, C., \& Aarsland, D. (2010). Health and wellbeing in care staff and their relations to organizational and psychosocial factors, care staff and resident factors in nursing homes. International Journal of Geriatric Psychiatry, 25(8), 789-797.

Van Bogaert, P., Dilles, T., Wouters, K., \& Van Rompaey, B. (2014). Practice environment, work characteristics and levels of burnout as predictors of nurse reported job outcomes, quality of care and patient adverse events: A study across residential aged care services. Open Journal of Nursing, 4, 343-355. https://doi.org/10.4236/ojn.2014.45040

Van den Brink, A. M. A., Gerritsen, D. L., de Valk, M. M. H., Oude Voshaar, R. C., \& Koopmans, R. (2017). Characteristics and health conditions of a group of nursing home patients with mental-physical multimorbidity the MAPPING study. International Psychogeriatrics, 29(6), 1037-1047. https://doi.org/10.1017/S1041610217000230

Wang, S., Liu, Y., \& Wang, L. (2015). Nurse burnout: Personal and environmental factors as predictors. International Journal of Nursing Practice, 21(1), 78-86. https://doi.org/10.1111/ijn.12216

Wynd, C. A. (2003). Current factors contributing to professionalism in nursing. Journal of Professional Nursing, 19(5), 251-261. https://doi. org/10.1016/S8755-7223(03)00104-2

Zimmerman, S., Williams, C. S., Reed, P. S., Boustani, M., Preisser, J. S., Heck, E., \& Sloane, P. D. (2005). Attitudes, stress, and satisfaction of staff who care for residents with dementia. The Gerontologist, 45(suppl 1), 96-105. https://doi.org/10.1093/geront/45.suppl_1.96

Zwijsen, S. A., Gerritsen, D. L., Eefsting, J. A., Smalbrugge, M., Hertogh, C. M., \& Pot, A. M. (2015). Coming to grips with challenging behaviour: A cluster randomised controlled trial on the effects of a new care programme for challenging behaviour on burnout, job satisfaction and job demands of care staff on dementia special care units. International Journal of Nursing Studies, 52(1), 68-74. https://doi.org/10.1016/j. ijnurstu.2014.10.003

How to cite this article: Collet J, de Vugt ME, Schols JMGA, Engelen GJJA, Winkens B, Verhey FRJ. Well-being of nursing staff on specialized units for older patients with combined care needs. J Psychiatr Ment Health Nurs. 2018;25:108-118. https://doi.org/10.1111/jpm.12445 